\title{
Tunable orbital angular momentum beams in the extreme ultraviolet/soft $\mathrm{x}$-ray regimes
}

Turpin, Alex, Rego, Laura, San Román, Julio, Picón, Antonio, Plaja, Luis, et al.

Alex Turpin, Laura Rego, Julio San Román, Antonio Picón, Luis Plaja, Carlos Hernández-García, "Tunable orbital angular momentum beams in the extreme ultraviolet/soft x-ray regimes," Proc. SPIE 10243, X-ray Lasers and Coherent X-ray Sources: Development and Applications, 102430V (17 May 2017); doi: 10.1117/12.2265811

SPIE. Event: SPIE Optics + Optoelectronics, 2017, Prague, Czech Republic 


\title{
Tunable orbital angular momentum beams in the extreme ultraviolet/soft $\mathrm{x}$-ray regimes
}

\author{
Alex Turpin ${ }^{\mathrm{a}, \mathrm{b}}$, Laura Rego ${ }^{\mathrm{c}}$, Julio San Román ${ }^{\mathrm{c}}$, Antonio Picón ${ }^{\mathrm{c}}$, Luis Plajac ${ }^{\mathrm{c}}$, and Carlos \\ Hernández-García ${ }^{c}$ \\ aDepartament de Física, Universitat Autònoma de Barcelona, E-08193 Bellaterra, Spain \\ ${ }^{\mathrm{b}}$ Center of Advanced European Studies and Research, 53175 Bonn, Germany \\ ${ }^{ }$Grupo de Investigación en Aplicaciones del Láser y Fotónica, Departamento de Física \\ Aplicada, University of Salamanca, E-37008, Salamanca, Spain
}

\begin{abstract}
High-order harmonic generation (HHG) has been recently proven to produce harmonic vortices carrying orbital angular momentum (OAM) in the extreme-ultraviolet (XUV) region from the nonlinear up-conversion of infrared vortex beams. In this work we present two methods to control and extend the OAM content of the harmonic vortices. First, we show that when a driver combination of different vortex modes is used, HHG leads to the production of harmonic vortices with a broad OAM content due to its nonperturbative nature. Second, we show that harmonic vortices with two discrete OAM contributions - so called fractional OAM modes- are generated when HHG is driven by conical refraction beams. Our work offers the possibility of generating tunable OAM beams in the XUV regime, potentially extensible to the soft $\mathrm{x}$ rays, overcoming the state of the art limitations for the generation of OAM beams far from the visible domain.
\end{abstract}

Keywords: Orbital angular momentum, extreme-ultraviolet, $\mathrm{X}$ rays, high harmonic generation, attosecond science, conical refraction.

\section{INTRODUCTION}

Our capability of harnessing light properties determines our limits to observe and control Nature at the extreme spatio-temporal scales. During the last decades, laser technology has boosted an ultrafast revolution, and nowadays it is possible to obtain coherent radiation from the infrared (IR) to the soft $\mathrm{x}$ rays, tailored as ultrashort light bursts. In this sense, the control over the temporal and spectral light properties has been indispensable to access the ultrafast and nanometer scales. In addition, topological light properties -such as polarization or orbital angular momentum (OAM) - have drawn the attention of the scientific community due to their ability to unveil new scenarios of laser-matter interaction, from the quantum to the macroscopic physics levels. In particular, twisted light beams carrying $\mathrm{OAM}^{1}$-so called optical vortices- offer a unique capability to harness light matterinteraction processes by adding a new degree of freedom over the control of light beams: the topological charge. Light vortices exhibit a transversal spiral-phase structure around the beam axis, thus having a well-defined OAM, which is characterized by the topological charge, $\ell$, i.e. the number of $2 \pi$-phase shifts along the azimuth of the light beam. These beams are routinely produced in the visible and IR regimes by using spatial light modulators, holograms or diffractive optical elements. ${ }^{2,3}$ They have been proved to be suitable for a wide range of applications, from optical communication, micromanipulation, phase-contrast microscopy, to quantum optics, among others. ${ }^{4}$

There is an increasing interest to bring OAM beams into the extreme-ultraviolet (XUV) and x-ray regimes, in order to extend their applications down to the ultrafast and nanometer scales. Although x-ray optics could be used to imprint angular momentum into XUV and x-ray light, in practice such optics are challenging to fabricate and have poor throughput and limited bandwidth. During the last five years, high harmonic generation (HHG) has emerged as a unique process to directly generate XUV/x-ray OAM beams through nonlinear up-conversion

Further author information: (Send correspondence to C.H.-G.)

C.H.-G.: E-mail: carloshergar@usal.es, Telephone: +34 923294678

X-ray Lasers and Coherent X-ray Sources: Development and Applications, edited by Annie Klisnick,

Carmen S. Menoni, Proc. of SPIE Vol. 10243, 102430V · (c) 2017 SPIE

CCC code: $0277-786 \mathrm{X} / 17 / \$ 18 \cdot$ doi: $10.1117 / 12.2265811$

Proc. of SPIE Vol. 10243 102430V-1 
of an intense IR vortex beam. ${ }^{5-9}$ HHG is a nonperturbative process that presents a dual nature. Microscopically, each atom in a gas target acts as a nano-collider: the intense IR laser field first detaches an electronic wavepacket, then accelerates it, and finally drives it back to its parent ion. Upon recombination, the kinetic energy acquired by the electronic wave-packet is released in terms of higher-frequency harmonics of the driving field. Macroscopically, the phase relationship between the harmonics radiated from each atom is crucial to determine not only the efficiency, but the spatio-temporal properties of the emitted coherent radiation. Interestingly enough, HHG is able to map some properties of the driving field into the higher frequency radiation. Thus, one can sculpt the properties of the harmonics through proper modifications of the driver. As a result, HHG stands as a clean and technologically simple method to provide flashes of light with three unique properties: (i) spectrally, HHG produces coherent broadband pulses extending from the XUV to the soft x rays; (ii) temporally, HHG radiation is emitted in the form of ultrashort pulses in the attosecond timescale; (iii) mechanically, HHG radiation can be sculpted in its topological properties, both spin (polarization) and orbital (phase rotation) angular momentum.

Since the first observation of XUV vortices driven by HHG ${ }^{5}$ there have been different studies to predict, observe and control their topological charge. ${ }^{10}$ When driven by a single mode IR vortex beam, OAM conservation leads to the generation of highly charged XUV vortices, with a topological charge being a multiple integer -the harmonic order- of the driving beam, following the simple rule $\ell_{q}=q \ell$ (being $\ell_{q}$ the topological charge of the q-th order harmonic, and $\ell$ that of the driving beam). This law was theoretically predicted in Ref. ${ }^{6}$ and experimentally observed in Refs. ${ }^{7,9}$ Very recent works have made use of two driving beams in a noncollinear HHG geometry to generate low-charge harmonic vortices with adjustable topological charge. ${ }^{7,11}$

In this contribution we report two different methods to generate XUV vortices with multiple-OAM content. First, we demonstrate that the nonperturbative behavior of HHG can be exploited to generate multiple-OAM vortices when using several driving IR vortices with different topological charge. ${ }^{12}$ We explore the underlying mechanisms for this phenomena and derive a general conservation rule for the non-perturbative OAM build-up. Second, we generate harmonic vortices with two OAM contributions -also called fractional OAM beams- when driving HHG by conical refraction (CR) beams. ${ }^{13}$ CR beams are obtained in optical biaxial crystals when a beam propagates parallel to one of the two optic axes, and they present fractional OAM. We show that the properties of CR-like beams can be transferred to the XUV regime through HHG. ${ }^{13}$

For the results reported in this contribution, we have performed simulations of HHG including propagation using the electromagnetic field propagator. ${ }^{14}$ The driving laser pulse exhibit a peak intensity at focus of $1.4 \times 10^{14}$ $\mathrm{W} / \mathrm{cm}^{2}$, with a central wavelength of $800 \mathrm{~nm}$. The rest of parameters can be found in Refs. ${ }^{12,13}$

\section{GENERATION OF XUV MULTI-MODE VORTICES}

Once the topological charge of XUV vortices driven by single-OAM IR beams was characterized, ${ }^{6,7,9}$ one may think that the use of a combination of two driving beams with different OAM components will results in the production of XUV vortices with several OAM contributions. In particular, let us consider a driving beam composed of two OAM modes, $\ell_{1}$ and $\ell_{2}$. From the photon picture, one can understand the OAM buildup of the $q$-th order harmonic from different absorption channels $(q-n, n), n$ ranging from 0 to $q$, associated with the two OAM modes of the driving field. This photon picture is similar to that previously considered in the conservation of linear momentum in noncollinear HHG schemes. ${ }^{15,16}$ Each channel $(q-n, n)$ has a weight proportional to the binomial probability distribution associated with the photon number combinations of absorbing $q-n$ photons from the $\ell_{1}$ mode and $n$ photons from the $\ell_{2}$ mode. As a consequence, the simple OAM buildup law $\left(\ell_{q}=q \ell\right)$ is converted into

$$
\ell_{q}=(q-n) \ell_{1}+n \ell_{2}
$$

However, we have found that the nonperturbative behavior of HHG modifies substantially this law, broadening the OAM spectrum of the XUV harmonic vortices produced. ${ }^{12}$ In the non-perturbative case, where one can consider that the harmonic amplitude scales with the $p$-th power of the fundamental field, the OAM buildup law is found to be: ${ }^{12}$

$$
\ell_{q}=(q-n) \ell_{1}+n \ell_{2}+m\left(\ell_{2}-\ell_{1}\right)
$$


where $n$ labels the harmonic channel, that now ranges from $(q-p) / 2$ to $(q+p) / 2$, being $p \sim 4$ for the harmonic in the plateau region. ${ }^{12}$ Note that there is a new term in the non-perturbative OAM buildup law, $m\left(\ell_{2}-\ell_{1}\right)$, where the index $m$ represents the azimuthal diffraction order induced by the intrinsic phase ${ }^{17,18}$ of the HHG process. ${ }^{12}$ This conservation rule collapses to the perturbative case -Eq. (1)- when $p \rightarrow q$ and $m=0$, and to the linear scaling law $\left(\ell_{q}=q \ell\right)$ found with single-OAM modes if $\ell_{1}=\ell_{2}=\ell$.

(a) HHG driven by a single-mode IR vortex
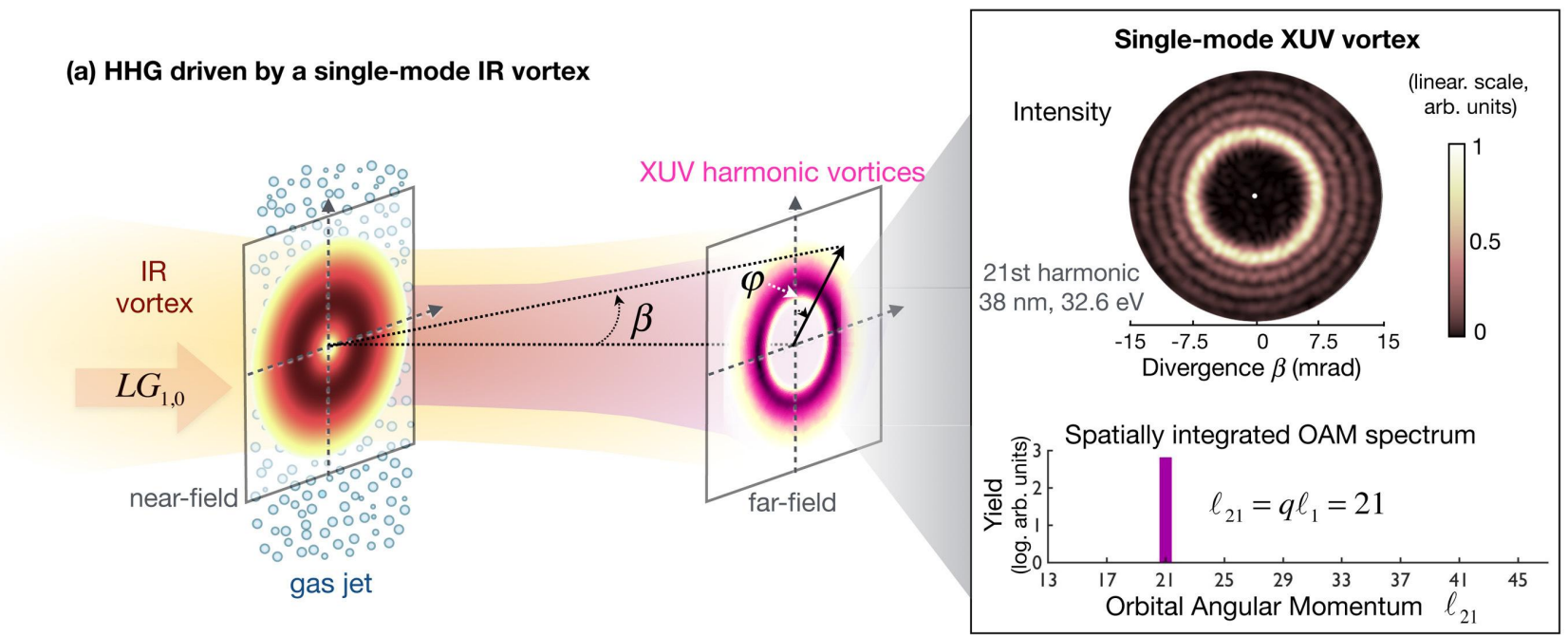

(b) HHG driven by a combination of two IR vortices
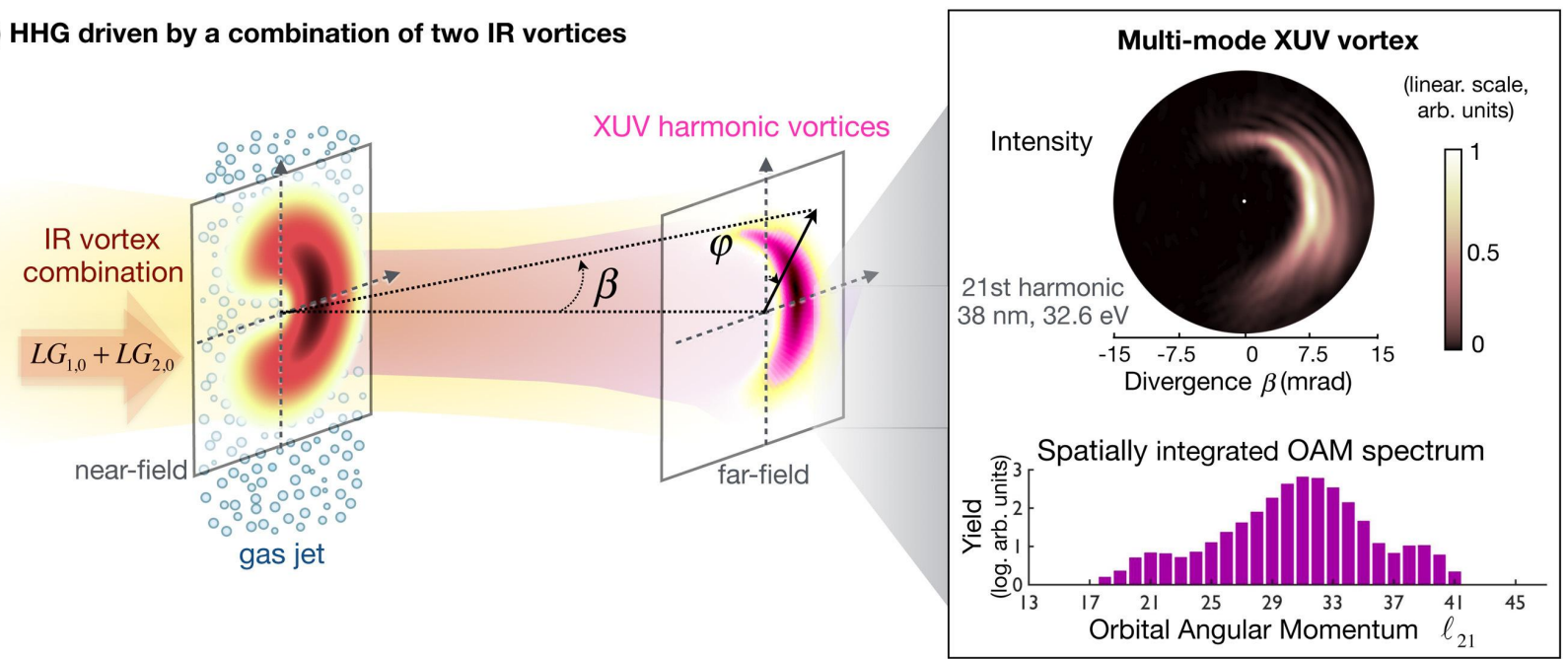

Figure 1. Schemes to produce single-OAM (a) and multiple-OAM (b) XUV harmonic vortices through high harmonic generation. In (a) a single-mode IR $(800 \mathrm{~nm})$ vortex beam with $\ell=1$ is focused into an argon gas jet, where high-order harmonics are produced in the form of XUV vortices. On the right panel we show the spatial intensity profile and OAM spectrum of the 21 st harmonic $(38 \mathrm{~nm}, 32.6 \mathrm{eV})$. A single OAM component is clearly generated, following the simple law $\ell_{21}=21 \ell=21$. In (b), the driving beam is composed of two IR vortex beams with $\ell_{1}=1$ and $\ell_{2}=2$. In this case, the OAM spectrum of the 21 st harmonic vortex is substantially broadened, exhibiting OAM contributions from $\ell_{21}=18$ to $\ell_{21}=42$, as depicted by Eq. (2).

In Fig. 1 we show the scheme to produce XUV vortices using HHG driven by (a) a single-OAM IR beam $(\ell=1)$, and (b) a driving combination of $\ell_{1}=1$ and $\ell_{2}=2$ modes, each one with the same weight. On the right we show the spatial intensity distribution and OAM spectrum of the 21st harmonic $(38 \mathrm{~nm}, 32.6 \mathrm{eV})$. Whereas in (a) the 21st harmonic vortex exhibit a single topological charge of $\ell_{21}=21$-as shown in previous works ${ }^{6,7,9}$-, in (b) the OAM spectrum is broadened, following the nonperturbative OAM build-up rule given by Eq. (2). ${ }^{12}$ 


\section{GENERATION OF XUV FRACTIONAL ORBITAL ANGULAR MOMENTUM BEAMS}

\subsection{Conical Refraction}

Conical refraction (CR) is a birefringent optical effect occurring in biaxial crystals when a light beam propagates parallel to one of its optic axis. ${ }^{19}$ For a focused Gaussian input beam, the CR beam evolves in free-space as a double-cone light structure, ${ }^{20}$ see Fig. 2(a). The CR beam is mainly characterized by its transverse intensity pattern at the otherwise expected focal plane, this pattern being formed by a pair of concentric bright rings split by a null intensity ring, as shown in Fig. 2(b). The state of polarization of the CR rings is linear at every point, with the azimuth rotating continuously along them satisfying that every two diametrically opposite points are orthogonally polarized, as depicted by the double white arrows in Fig. 2(b).

To understand the origin of the involved CR polarization distribution, note that the CR beam is formed by the interference of two Bessel-like functions, called as $B_{0}$ and $B_{1}{ }^{19,21,22}$ with similar free-space beam evolution [see Figs. 2(d)-(h)] but different angular momentum (both spin and orbital components). For a circularly polarized input beam described in the basis $\mathbf{e}_{ \pm}=(1, \pm i) / \sqrt{2}$ ( + for right-handed and - for left-handed), the electric field behind the crystal becomes:

$$
\mathbf{E}_{ \pm}=B_{0} \mathbf{e}_{ \pm}+B_{1} e^{ \pm i \varphi} \mathbf{e}_{\mp}
$$

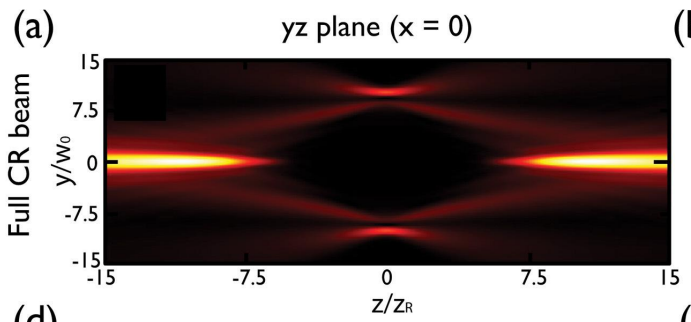

(b) Focal xy plane $(z=0)$

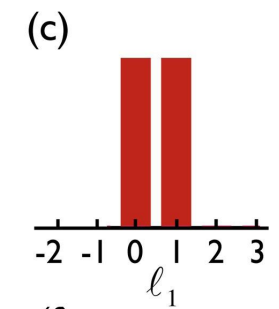

(d)
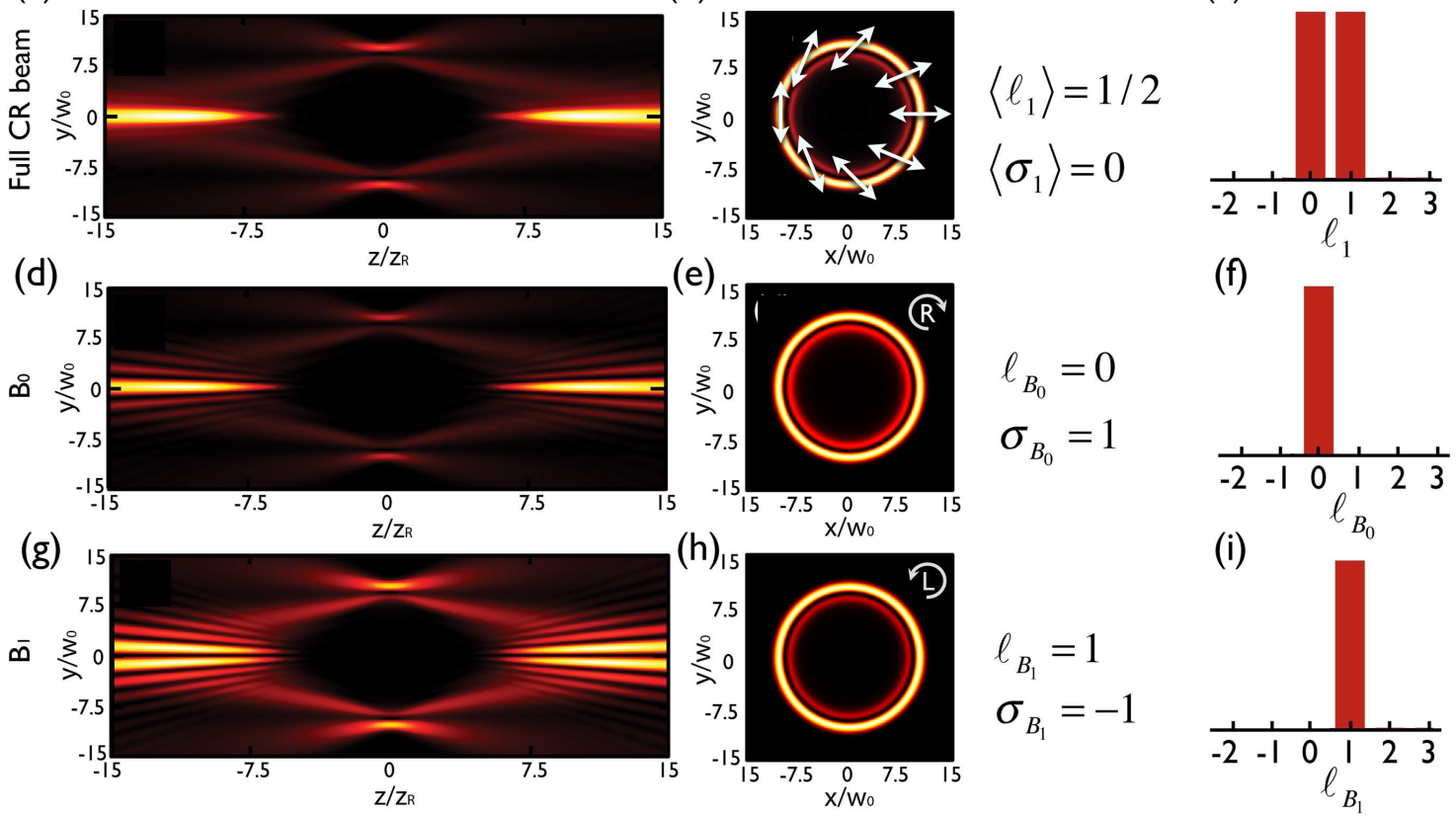

Figure 2. Conical refraction for a right-handed circularly polarized Gaussian input beam. Images (a), (d), and (g) are cuts of the intensity pattern in the $y z$ plane at $x=0$ showing the space evolution of the CR beam, the $B_{0}$ component, and the $B_{1}$ component respectively. Images (b), (e), and (h) show the transverse intensity distribution obtained at the focal plane of the CR beam, the $B_{0}$ component, and the $B_{1}$ component, respectively. Panels (c), (f), and (i) show, respectively, the OAM spectrum of the whole CR beam, the $B_{0}$ component, and the $B_{1}$ component.

In Figs. 2(c), (f), and (i) we show the OAM spectrum of the whole CR beam, the $B_{0}$ component, and the $B_{1}$ component, respectively, for a right-handed circularly polarized input beam. According to Eq. (3), the $B_{0}$ beam preserves the state of polarization of the input beam and possesses null OAM, i.e. it possesses spin angular momentum $\sigma_{B_{0}}= \pm 1$ and orbital angular momentum $l_{B_{0}}=0$. In contrast, the $B_{1}$ beam is orthogonally polarized to the input beam (thus $\sigma_{B_{1}}=\mp 1$ ) and carries one unit of OAM per photon $\left(l_{B_{1}}= \pm 1\right.$ ), due to the spin-orbit coupling introduced by the biaxial crystal, ${ }^{23,24}$ manifesting as an on-axis null intensity point preserved upon propagation, see Fig. $2(\mathrm{~g})$. As a consequence, the global CR beam possesses $\pm \hbar / 2$ OAM per photon. ${ }^{23-27}$ 


\subsection{High harmonic generation driven by conical refraction beams}

The physical scenario considered here is analogous to the one for the generation of XUV multimode vortices presented in Section 2, see Fig 3(a). A CR beam generated with a biaxial crystal is focused onto a gas jet, ensuring that the double-ring $\mathrm{CR}$ structure obtained at the focal plane of the beam hits the gas jet. As in Section 2, the IR beam composed by different OAM contributions: $l_{B_{0}}=0$ and $l_{B_{1}}=1$ [see Fig. 3(b)], where we have considered right-handed circularly polarized input light sent to the biaxial crystal, i.e., the + sign in Eq. (3). However, we recall that in this case the $B_{0}$ and $B_{1}$ components have opposite spin angular momentum: $\sigma_{B_{0}}=1, \sigma_{B_{1}}=-1$. As a consequence, in this HHG scheme the conservation of both OAM and SAM have to be taken into account. As we show in what follows, this mutual conservation of OAM and SAM leads to the generation of HHG beams with fractional OAM.

(a) High harmonic generation driven by conical refraction beams

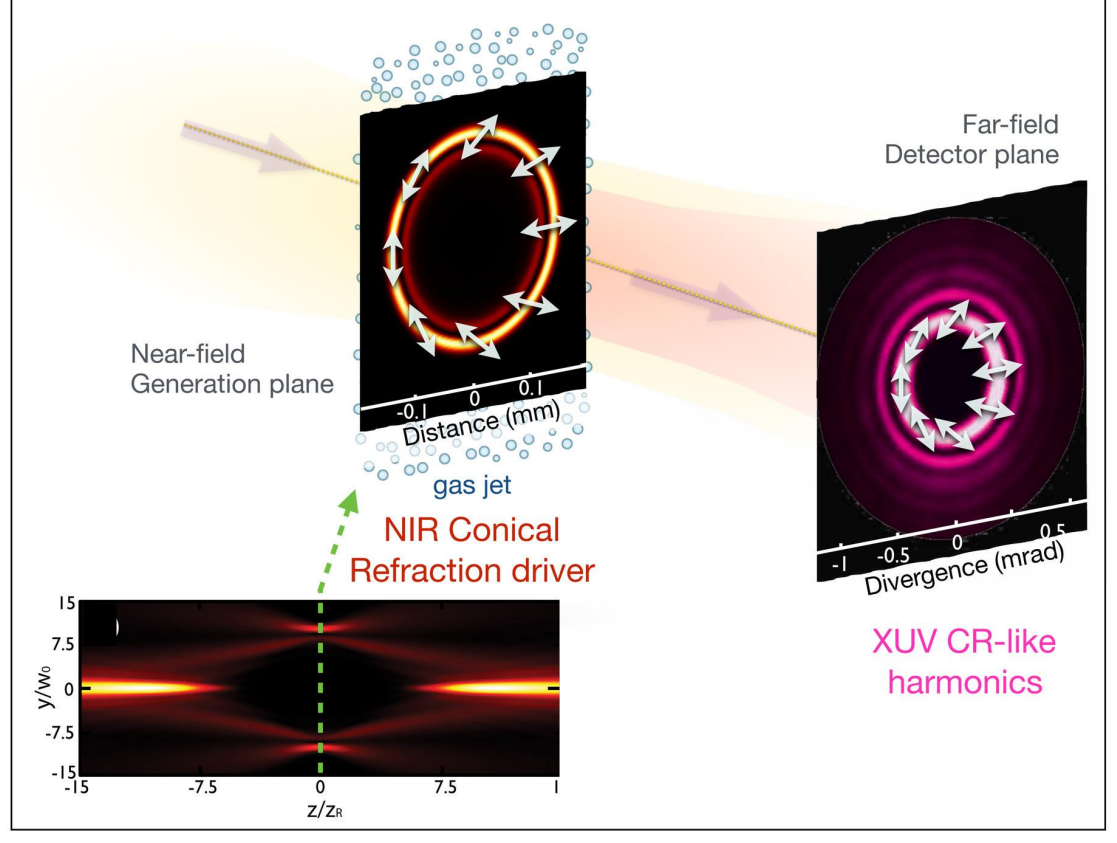

OAM spectrum of the IR fundamental and XUV harmonics (b) (S) D
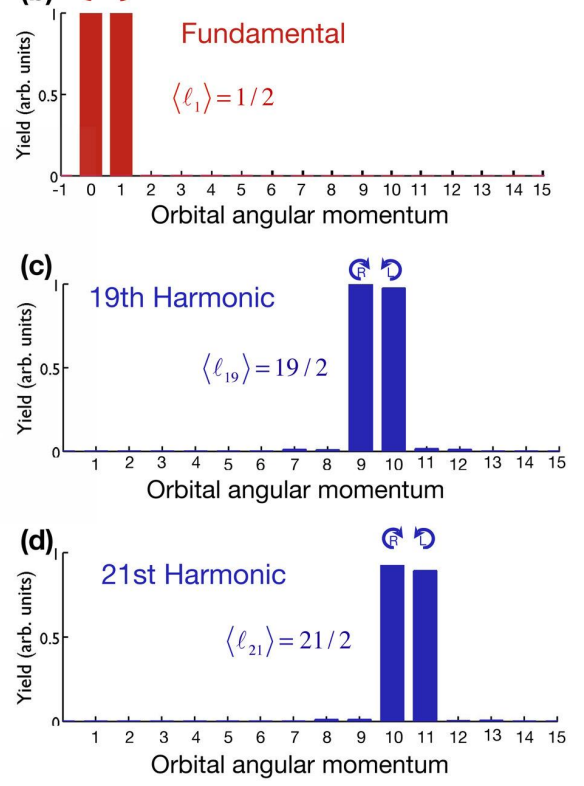

Figure 3. (a) Scheme of high harmonic generation driven by conical refraction beams to produce XUV fractional-OAM vortices: an intense IR CR beam generated with a biaxial crystal is focused onto a gas jet. The left inset shows the CR beam intensity distribution at the focal plane $(\mathrm{z}=0$ in the bottom inset, where the transverse intensity profile of the CR beam along the propagation axis is shown). The linear polarization distribution (depicted with white arrows) rotates along the azimuth. High-order harmonics are generated in each atom of the gas jet, and then propagated towards a far-field detector. The right inset shows the far-field HHG intensity and polarization distributions, which retain the CR-like beam structure. In the right column we show the OAM spectrum of the fundamental beam, (c) the 19th harmonic, and (d) the 21st harmonic. The two components of the fundamental CR beam can be distinguished: the $B_{0}$ beam $\left(\ell_{B_{0}}=0\right.$, $\left.\sigma_{B_{0}}=+1\right)$, and the $B_{1}$ beam $\left(\ell_{B_{1}}=1, \sigma_{B_{1}}=-1\right)$. Thus, the fundamental beam exhibits fractional-OAM $\left\langle\ell_{1}\right\rangle=1 / 2$. The high-order harmonics present analogously two OAM contributions with counter-rotating polarization. Angular momentum conservation conveys the generation of fractional-OAM harmonics with topological charge $\left\langle\ell_{19}\right\rangle=19 / 2$, and $\left\langle\ell_{21}\right\rangle=21 / 2$.

From the photon picture one can consider the generation of the $q$-th order harmonic from the absorption of $n_{0}$ and $n_{1}$ photons from the $B_{0}$ and $B_{1}$ beams respectively, conveying the absorption channel $\left(n_{0}, n_{1}\right)$, and with corresponding energy $q=n_{0}+n_{1}$. Parity conservation forces $n_{0}+n_{1}$ to be an odd integer while the restriction over the photon spin $(|\sigma|=1)$ implies that $n_{1}=n_{0} \pm 1$, i.e., the number of photons absorbed from the $B_{0}$ and $B_{1}$ beams only differs by one. Therefore, only two possible channels generating $q$-th order harmonic can be found: the R (right-handed circularly polarized) channel $\left(\frac{q-1}{2}, \frac{q+1}{2}\right)$, and the L (left-handed circularly polarized) 
channel $\left(\frac{q+1}{2}, \frac{q-1}{2}\right)$. If we analyze angular momentum conservation in its separate spin and orbital components, on the one hand, from SAM conservation of each of the R and $\mathrm{L}$ channels leads to

$$
\begin{aligned}
\sigma_{q, R} & =\frac{q+1}{2} \sigma_{B_{0}}+\frac{q-1}{2} \sigma_{B_{1}}=1 \\
\sigma_{q, L} & =\frac{q-1}{2} \sigma_{B_{0}}+\frac{q+1}{2} \sigma_{B_{1}}=-1
\end{aligned}
$$

On the other hand, OAM conservation of each separate channel implies the following build-up rules

$$
\begin{aligned}
& \ell_{q, R}=\frac{q+1}{2} \ell_{B_{0}}+\frac{q-1}{2} \ell_{B_{1}}=\frac{q-1}{2} \\
& \ell_{q, L}=\frac{q-1}{2} \ell_{B_{0}}+\frac{q+1}{2} \ell_{B_{1}}=\frac{q+1}{2} .
\end{aligned}
$$

Finally, note that HHG is restricted to linearly polarized drivers due to the reduced efficiency of the HHG process with the ellipticity of the driving beam in single-color schemes, such as as the one presented in this work. Therefore, both channels $\mathrm{R}$ and $\mathrm{L}$ are forced to be present with similar efficiency, yielding to a net absorption of linearly polarized photons. As a consequence, the resulting $q$-th order harmonic exhibits linear polarization and fractional-OAM,

$$
\begin{aligned}
\left\langle\sigma_{q}\right\rangle & =0 \\
\left\langle\ell_{q}\right\rangle & =\frac{q}{2}
\end{aligned}
$$

In Fig. 3 and it can be observed that the 19th [panel (c)] and 21st [panel (d)] harmonics present two OAM contributions with counter-rotating polarization, corresponding to the $\mathrm{R}$ and $\mathrm{L}$ channels described previously, and fractional-OAM of $\left\langle\ell_{19}\right\rangle=19 / 2$ and the $\left\langle\ell_{21}\right\rangle=21 / 2$, respectively, as predicted above.

\section{CONCLUSIONS AND OUTLOOK}

In this contribution we have reviewed two different methods to generate XUV vortices with different OAM content. Both methods are based on up-converting intense IR OAM beams into higher-frequency OAM beams through high harmonic generation. On one hand, we have shown that when HHG is driven by a combination of IR vortices with different topological charge, multi-mode XUV harmonics with a broad OAM content are naturally produced due to the nonperturbative behavior of HHG. ${ }^{12}$ A new OAM build-up law that takes into account the nonperturbative nature of HHG has been presented. On the other hand, if HHG is driven by IR conical refraction beams, fractional-OAM harmonics (with two well-defined OAM contributions) are generated. ${ }^{13}$ This scheme is particularly interesting as both spin and angular momentum conservation have to be taken into account.

Whereas in this work we have presented harmonic vortices in the XUV regime extending up to tens of eV, it seems that there is no intrinsic limit that precludes their extension to the soft x-ray regime, by, for example, using mid-infrared driving beams, ${ }^{28}$ or intense vacuum-ultraviolet drivers. ${ }^{29}$

Finally, one should note that XUV vortices generated through HHG are particularly interesting from the temporal point of view. For instance, single-mode XUV vortex generation through HHG leads to the production of helical attosecond beams ${ }^{6,9}$-also called "attosecond light springs". ${ }^{30}$ These are the result of the composition of several harmonic vortices with different topological charge. The temporal and spatial properties of these helical attosecond beams can be modified through proper modification of macroscopic phase-matching conditions..$^{8,31}$ If HHG is driven by conical refraction beams, several fractional-OAM harmonic vortices can be synthesized to generate structured attosecond light springs, i.e., attosecond helical beams with varying linear polarization along the azimuth. The generation of XUV beams that exhibit varying polarization ${ }^{32}$ and OAM may open the route for ultrafast studies of spin to orbit angular momentum exchange in at the nanometer scale. ${ }^{33,34}$ 


\section{ACKNOWLEDGMENTS}

C.H.-G. acknowledges support from the Marie Curie International Outgoing Fellowship within the EU Seventh Framework Programme for Research and Technological Development (2007-2013), under REA grant Agreement No. 328334. C.H.-G., and J.S.-R. acknowledge support and from Junta de Castilla y León (Project SA046U16) and MINECO (FIS2013-44174-P, FIS2016-75652-P). A.T. acknowledges support from the MECD (AP2010-2310), the MINECO (FIS2011-23719), the Catalan government (SGR2014-1639), and the Max Planck Society. A.P. acknowledges support from the European Unions Horizon 2020 research and innovation programme under the Marie Sklodowska-Curie grant agreement No. 702565.

\section{REFERENCES}

[1] Allen, L., Beijersbergen, M. W., Spreeuw, R. J. C., and Woerdman, J. P., "Orbital angular momentum of light and the transformation of laguerre-gaussian laser modes," Phys. Rev. A 45, 8185-8189 (1992).

[2] Atencia, J., Collados, M.-V., Quintanilla, M., Marín-Sáez, J., and Sola, I. J., "Holographic optical element to generate achromatic vortices," Opt. Express 21(18), 21056-21061 (2013).

[3] Sola, I., Collados, V., Plaja, L., Méndez, C., San Román, J., Ruiz, C., Arias, I., Villamarín, A., Atencia, J., Quintanilla, M., and Roso, L., "High power vortex generation with volume phase holograms and non-linear experiments in gases," Applied Physics B 91(1), 115-118 (2008).

[4] Torres, J. and Torner, L., [Twisted Photons: Applications of Light with Orbital Angular Momentum], Wiley (2011).

[5] Zürch, M., Kern, C., Hansinger, P., Dreischuh, A., and Spielmann, C., "Strong-field physics with singular light beams," Nature Physics 8(10), 743-746 (2012).

[6] Hernández-García, C., Picón, A., San Román, J., and Plaja, L., "Attosecond extreme ultraviolet vortices from high-order harmonic generation," Phys. Rev. Lett. 111, 083602 (2013).

[7] Gariepy, G., Leach, J., Kim, K. T., Hammond, T. J., Frumker, E., Boyd, R. W., and Corkum, P. B., "Creating high-harmonic beams with controlled orbital angular momentum," Phys. Rev. Lett. 113, 153901 (2014).

[8] Hernández-García, C., San Román, J., Plaja, L., and Picón, A., "Quantum-path signatures in attosecond helical beams driven by optical vortices," New Journal of Physics 17(9), 093029 (2015).

[9] Géneaux, R., Camper, A., Auguste, T., Gobert, O., Caillat, J., Taieb, R., and Ruchon, T., "Synthesis and characterization of attosecond light vortices in the extreme ultraviolet," Nat. Commun. 7, 12583 (2016).

[10] Hernández-García, C., "A twist in coherent X rays," Nature Physics 13 (2017).

[11] Gauthier, D., Ribic, P. R., Adhikary, G., Camper, A., Cucini, C. C. R., DiMauro, L. F., Dovillaire, G., Frassetto, F., Géneaux, R., Miotti, P., Poletto, L., Ressel, B., Spezzani, C., Stupar, M., Ruchon, T., and Ninno, G. D., "Tunable orbital angular momentum in high-harmonic generation," arXiv:1608.06907 .

[12] Rego, L., Román, J. S., Picón, A., Plaja, L., and Hernández-García, C., "Nonperturbative twist in the generation of extreme-ultraviolet vortex beams," Phys. Rev. Lett. 117, 163202 (2016).

[13] Turpin, A., Rego, L., Picón, A., San Román, J., and Hernández-García, C., "Extreme Ultraviolet Fractional Orbital Angular Momentum Beams from High Harmonic Generation," Sci. Rep. 7, 43888 (2017).

[14] Hernández-García, C., Pérez-Hernández, J. A., Ramos, J., Jarque, E. C., Roso, L., and Plaja, L., "Highorder harmonic propagation in gases within the discrete dipole approximation," Phys. Rev. A 82, 033432 (2010).

[15] Bertrand, J. B., Wörner, H. J., Bandulet, H.-C., Bisson, E., Spanner, M., Kieffer, J.-C., Villeneuve, D. M., and Corkum, P. B., "Ultrahigh-order wave mixing in noncollinear high harmonic generation," Phys. Rev. Lett. 106, 023001 (2011).

[16] Hickstein, D. D., Dollar, F. J., Grychtol, P., Ellis, J. L., Knut, R., Hernández-García, C., Zusin, D., Gentry, C., Shaw, J. M., Fan, T., Dorney, K. M., Becker, A., Jaron-Becker, A., Kapteyn, H. C., Murnane, M. M., and Durfee, C. G., "Non-collinear generation of angularly isolated circularly polarized high harmonics," Nature Photonics 9(11), 743-750 (2015).

[17] Corkum, P. B., "Plasma perspective on strong field multiphoton ionization," Phys. Rev. Lett. 71, 1994-1997 (1993). 
[18] Hernández-García, C. and Plaja, L., "Off-axis compensation of attosecond pulse chirp," Journal of Physics B: Atomic, Molecular and Optical Physics 45(7), 074021 (2012).

[19] Turpin, A., Loiko, Y. V., Kalkandjiev, T. K., and Mompart, J., "Conical refraction: fundamentals and applications," Laser Photon. Rev. 10, 750-751 (2016).

[20] Turpin, A., Loiko, Y. V., Kalkandjiev, T. K., Tomizawa, H., and Mompart, J., "On the dual-cone nature of the conical refraction phenomenon," Opt. Lett. 40, 1639-1642 (2015).

[21] Berry, M. V., "Conical diffraction asymptotics: fine structure of poggendorff rings and axial spike," J. Opt. A: Pure and App. Opt. 6, 289-300 (2004).

[22] Phelan, C. F., O'Dwyer, D. P., Rakovich, Y. P., Donegan, J. F., and Lunney, J. G., "Conical diffraction and bessel beam formation with a high optical quality biaxial crystal," Opt. Express 17, 12891-12899 (2009).

[23] Berry, M. V., Jeffrey, M. R., and Mansuripur, M., "Orbital and spin angular momentum in conical diffraction," J. Opt. A: Pure and App. Opt. 7, 685-690 (2005).

[24] Turpin, A., Loiko, Y. V., Peinado, A., Lizana, A., Kalkandjiev, T. K., Campos, J., and Mompart, J., "Polarization tailored novel vector beams based on conical refraction," Opt. Express 23, 5704-5715 (2015).

[25] O'Dwyer, D. P., Phelan, C. F., Rakovich, Y. P., Eastham, P. R., and Lunney, J. Gand Donegan, J. F., "Generation of continuously tunable fractional optical orbital angular momentum using internal conical diffraction.," Opt. Express 18, 16480-16485 (2010).

[26] Ballantine, K. E., Donegan, J. F., and Eastham, P. R., "There are many ways to spin a photon: Halfquantization of a total optical angular momentum," Sci. Adv. 2, e1501748 (2016).

[27] Peet, V., "Conical refraction in a degenerated two crystal cascade," J. Opt. 18, 015607 (2016).

[28] Popmintchev, T., Chen, M.-C., Popmintchev, D., Arpin, P., Brown, S., Ališauskas, S., Andriukaitis, G., Balčiunas, T., Mücke, O. D., Pugzlys, A., Baltuška, A., Shim, B., Schrauth, S. E., Gaeta, A., HernándezGarcía, C., Plaja, L., Becker, A., Jaron-Becker, A., Murnane, M. M., and Kapteyn, H. C., "Bright coherent ultrahigh harmonics in the kev x-ray regime from mid-infrared femtosecond lasers," Science 336(6086), 1287-1291 (2012).

[29] Popmintchev, D., Hernández-García, C., Dollar, F., Mancuso, C., Pérez-Hernández, J. A., Chen, M.-C., Hankla, A., Gao, X., Shim, B., Gaeta, A. L., Tarazkar, M., Romanov, D. A., Levis, R. J., Gaffney, J. A., Foord, M., Libby, S. B., Jaron-Becker, A., Becker, A., Plaja, L., Murnane, M. M., Kapteyn, H. C., and Popmintchev, T., "Ultraviolet surprise: Efficient soft x-ray high-harmonic generation in multiply ionized plasmas," Science 350(6265), 1225-1231 (2015).

[30] Pariente, G. and Quéré, F., "Spatio-temporal light springs: extended encoding of orbital angular momentum in ultrashort pulses," Opt. Lett. 40(9), 2037-2040 (2015).

[31] Hernández-García, C., Rego, L., San Román, J., Picón, A., and Plaja, L., "Attosecond twisted beams from high-order harmonic generation driven by optical vortices," High Power Laser Science and Engineering 5, e3 (2017).

[32] Hernández-García, C., Turpin, A., Román, J. S., Picón, A., Drevinskas, R., Cerkauskaite, A., Kazansky, P. G., Durfee, C. G., and Sola, I. J., "Extreme ultraviolet vector beams driven by infrared lasers," Optica, in press (2017).

[33] Bliokh, K. Y., Rodríguez-Fortuño, F. J., Nori, F., and Zayats, A. V., "Spin-orbit interactions of light," Nature Photonics 9(12), 796-803 (2015).

[34] Cardano, F. and Marrucci, L., "Spin-orbit photonics," Nature Photonics 9(12), 776-778 (2015). 